\title{
Comparative Study of Solid Phase Adsorption Sampling Tubes for the Analysis of Acrolein in Ambient Air
}

\author{
Chen Shen \\ Dept. of Environmental and Biological Engineering, \\ Nanjing University of Science and Technology, \\ Nanjing, China \\ shen0703270110@163.com
}

\begin{abstract}
Two kinds of sampling tubes have been developed for the analysis of acrolein in ambient air, which were filled with $200 \mathrm{mg}$ silica gel sorbent coated with either pentafluorophenyl hydrazin (PFPH) or 2,4-dinitrophenyl hydrazine(DNPH). GC/EDC and HPLC/UV are employed to evaluate the performance of PFPH-coated and DNPH-coated tubes, respectively. The PFPH or DNPH coating amount of each tube, the sampling flow rate and the storage time were optimized to conduct a comparative study about the extraction efficiency $(96 \%$ for PFPH, 92\% for DNPH), collection efficiency $(98 \%$ for PFPH, $99 \%$ for DNPH), storage capacity $(5 \mathrm{~d}$ for PFPH, less than $5 \mathrm{~h}$ for DNPH) of both.
\end{abstract}

\section{Keywords - sampling tubes acrolein PFPH DNPH}

\section{I .INTRODUCTION}

Among all the volatile compounds, carbonyls are an important class with highly reactive and adverse effects on human health, which have drawn extensive attentions and interests[1]. These significantly reactive compounds, namely formaldehyde, acetaldehyde, acrolein, can certainly cause serious and irreparable damage on biological substances such as proteins, phospholipids, and DNA[2]. Acrolein, in particular, at high acute exposures, can induce oxidative stress and delayed-onset lung injury, including asthma, congestion, and decreased pulmonary function. In addition, it is proved to

\footnotetext{
*Corresponding author. Tel. 86-25-84315521

E-mail address:wzp306@sina.com
}

\author{
Zhengping Wang*, Lina Luo \\ Dept. of Environmental and Biological Engineering, \\ Nanjing University of Science and Technology, \\ Nanjing, China \\ wzp306@sina.com, 523354978@qq.com
}

show mutagenic and genotoxic properties . The main sources of acrolein in the air are the following three aspects: direct combustion[3](e.g., forest fire); oxidation of hydrocarbons in the natural and social activities[4](e.g., primary photooxidation of 1,3-butadiene); incomplete combustion[5](e.g., smoke, cooking, wood burning, forest fires, burning incense, motor vehicle emissions). However, due to trace or ultra-trace levels of acrolein in the atmosphere, it is urgent to establish a reliable and effective acrolein sampling method as a prerequisite for the detection. DNPH-coated solid sorbent sampling methods for carbonyls have been extensively evaluated and promoted to present day[6,7]. Also, latest researches have proven that pentafluorophenyl hydrazine (PFPH) is a better derivative agent than DNPH due to its higher stability and volatility $[8,9]$. However, a large number of these method evaluations were limited to formaldehyde. In this paper, two kinds of sampling tubes filled with PFPH-coated or DNPH-coated silica gel were developed. The experiment involved the preparation of these two sampling tubes, the sampling process in a gas chamber under different conditions, detailed analysis and comparison.

\section{EXPERIMENT}

\section{A. Materials and Chemical agents}

Acrolein was purchased from Xi Ya Reagents Corporation. Acetonitrile(ACN), hexane were from CNPGC, $\mathrm{PFPH}(>98 \%)$ was from TCI, 2,4-DNPH( $>99 \%$ HPLC) was from Aladdin. Silica gel (60/80mush) and glass wool were from Shanghai ANPEL Scientific Instrument Co, Ltd. 


\section{B. PFPH-coated sampling tubes}

The sampling tube was made of glass $(8.0 \mathrm{~cm}$ length, 6 $\mathrm{mm}$ i.d. and $8 \mathrm{~mm}$ o.d.) with opening ends. Both glass wool and silica gel had been soaked with sulfuric acid overnight, and washed by deionized water to neutral, finally baked at $300^{\circ} \mathrm{C}$ for $3 \mathrm{~h}$ in a muffle so as to remove impurities that might have been adsorbed during their manufacturing process, transportation and storage. Glass tubes were repeatedly washed with deionized water and then dried at $70^{\circ} \mathrm{C}$. The PFPH coating agent was prepared in hexane at a concentration of $0.5 \mathrm{mg} / \mathrm{mL}$. The sorbent was prepared by mixing silica gel with PFPH coating agent, sealed in a container and placed at room temperature for more than 6 hours to ensure fully-coated with PFPH. After that, the coated silica gel was dried under a gentle gas stream of high-purity nitrogen. PFPH-coated silica was packed into separate part of the tube, $150 \mathrm{mg}$ in the front and $50 \mathrm{mg}$ in the back. Both ends and middle of the two parts were plugged with glass wools. Specially, the tubes were then wrapped with rubber caps coated with vacuum grease to avoid any contamination in the air, sealed in a Teflon bag and stored in refrigerator until use.

\section{DNPH-coated Sampling Tubes}

With the same pretreatment of glass tube, glass wool and silica gel. DNPH coating agent was prepared in acidified $\mathrm{ACN}$ at a concentration of $0.5 \mathrm{mg} / \mathrm{mL}$. Other processes were the same with the former.

\section{Sample Collection and Extraction}

A simply-constructed acrolein generation chamber was designed to evaluate the two tubes. Trace amount of acrolein (prepared in $\mathrm{ACN}$ at the concentration of $20 \mu \mathrm{L} / \mathrm{mL}$ ) was injected into the system to volatilize moderately throughout the space. Then acrolein was collected by drawing air in the chamber through the sampling tube with a personal sampling pump at different flow rates. The sampling time was $20 \mathrm{~min}$. Then the tubes were divided into various groups for analysis at once or storage. silica gel in each part of the sampled tube was eluted and analyzed separately. Notably, if the acrolein collected on the back part was $20 \%$ larger than that of the front part, the sample should be regarded as invalid. Those stored sample tubes should be analyzed within one week.

\section{E. Sample Analysis}

The PFPH-derivative was analyzed by GC/ECD (VarineCP-3800, CD-210 column, 30m*0.25mm*0.25 $\mu \mathrm{m}$ ) while the DNPH-derivative by HPLC/UV (Waters, XTerra

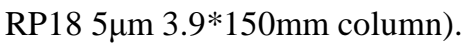

\section{RESULTS AND DISCUSSION}

\section{A. Solvent Extraction Efficiency}

For the PFPH-coated tubes, four samples of different concentration were selected to evaluate solvent extraction efficiency. Firstly, each sampled tube was extracted with $3 \mathrm{~mL}$ hexane, and then analyzed by GC/ECD. The extraction efficiency was calculated using the Eq.1

$$
\mathrm{EE}=100 \%\left(1-\mathrm{E}_{2} / \mathrm{E}_{1}\right) .
$$

where $E_{1}$ and $E_{2}$ were the acrolein amount of the first and second extracts. Hexane has been reported as an ideal extraction. However, according to the results shown in Fig.1, it is obvious that the higher concentration of acrolein, the lower EE is achieved. With further research, ACR-PFPH was found almost entirely readsorpted onto the silica gel within 5 hours, indicating a poor extraction capacity of haxane. Then in this paper, hexane was replaced by ACN. Fig.1 shows the EEs for ACR-PFPH eluted by ACN. The mean EE of ACR-PFPH is above $96 \%$. For the ACR-DNPH extracted by acidified ACN and analyzed by HPLC/UV, EE is above $92 \%$, slightly lower than the former.

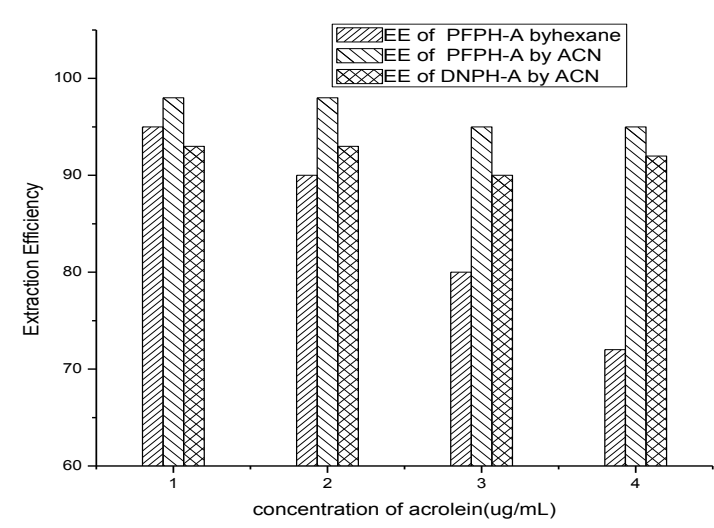

Fig. 1. Extraction Efficiency of the sampling tubes

\section{B. Sample Collection Efficiency}

Before evaluating any $\mathrm{CE}$ for each sampling tube, an 
appropriate sampling flow rate and acrolein concentrations in the chamber were estimated based on molar stoichiometry. This would make sufficient collection of acrolein available. The collection efficiency was calculated using the Eq.2,

$$
\mathrm{CE}=100 \%\left(1-\mathrm{A}_{\mathrm{b}} / \mathrm{A}_{\mathrm{f}}\right)
$$

where $A_{f}$ and $A_{b}$ were the acrolein collected on the front and back part of each tube. Acrolein of various concentration levels, DNPH or PFPH coating amount and sampling flow rate were considered as the key parameters to affect the $\mathrm{CE}$. In this study, three acrolein concentrations in the chamber $(0.1 \mu \mathrm{g} / \mathrm{L}, 0.2 \mu \mathrm{g} / \mathrm{L}, 0.5 \mu \mathrm{g} / \mathrm{L})$, four coating amounts $(0.2 \mathrm{mg}$, $0.3 \mathrm{mg}, 0.4 \mathrm{mg}, 0.5 \mathrm{mg}$ per $200 \mathrm{mg}$ silica gel) and four flow $\operatorname{rates}(200,300,500,800 \mathrm{~mL} / \mathrm{min})$ were tested.

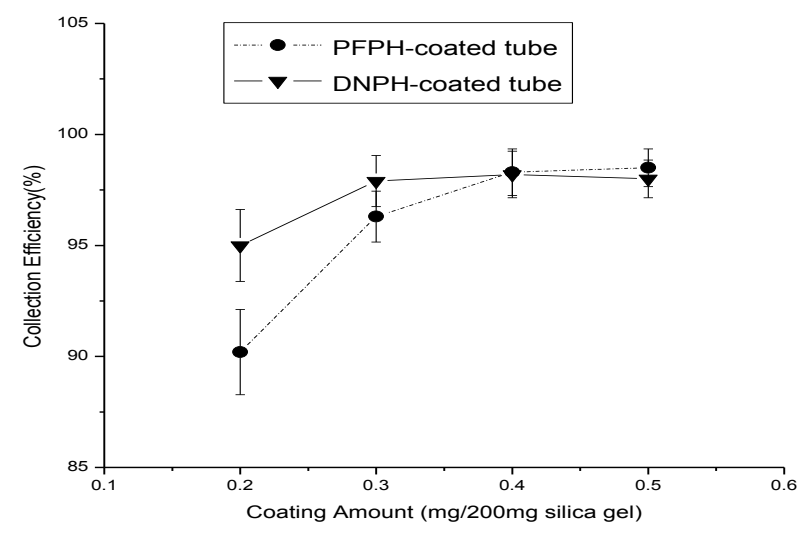

Fig. 2. CE versus different coating amount

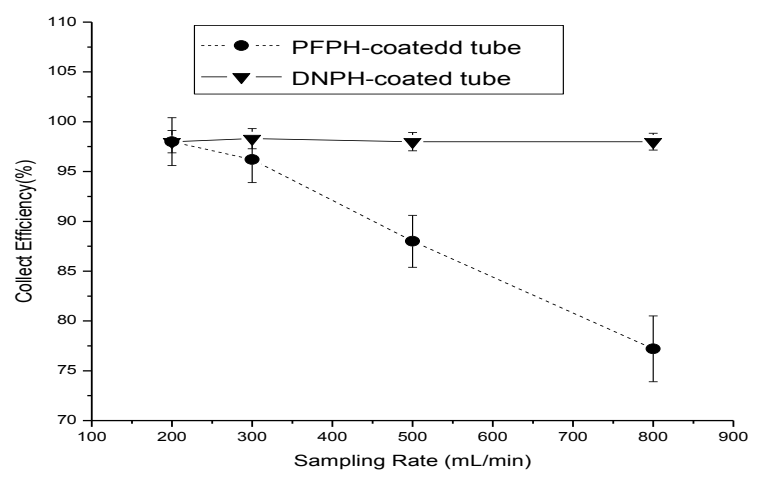

Fig. 3. CE versus different sampling rate

Fig.2 shows the CE of acrolein collected at three PFPH coating amounts at a sampling flow rate of $200 \mathrm{~mL} / \mathrm{min}$. It was obvious that the more derivatization coating, the better $\mathrm{CE}$ was achieved. In order to develop an inexpensive and efficient tube, we made the PFPH coating amount of $0.4 \mathrm{mg}$ per $200 \mathrm{mg}$ silica gel, and $0.3 \mathrm{mg}$ per $200 \mathrm{mg}$ silica gel for DNPH the most appropriate, where CE were maintaining above $98 \%$ for both. Fig. 3 shows the CE of various flow rates, PFPH-coated tubes gained high and almost the same CE of over $96 \%$ from $200-300 \mathrm{~mL} / \mathrm{min}$. However, when it was up to $500 \mathrm{~mL} / \mathrm{min}$, a slight decline was occured. While DNPH-coated tubes were almost all above $96 \%$ at four flow rates. In conclusion, the flow rate was $300 \mathrm{~mL} / \mathrm{min}$ for PFPH-coated tubes and $800 \mathrm{~mL} / \mathrm{min}$ for DNPH-coated tubes. In the whole test, the varied acrolein concentration showed little influence on the $\mathrm{CE}$, which can be ignored in later tests. The lower coating amount and higher flow rate of DNPH were due to the faster derivative reaction between acrolein and DNPH.

\section{Blank Levels and Storage Tests}

To further evaluate the sampling tubes, blank levels and sample storage duration were tested. 20 tubes of each kind were prepared, among which 5 tubes were used as blank levels. The rest 15 tubes were spiked with known amount of acrolein $(1 \mu \mathrm{g})$ and then divided into three groups and kept at either room temperature, in a refrigerator, or in a freezer. Notably, all tubes should be wrapped with rubber caps coated with vacuum grease, sealed with PTFE tape at both ends, finally put in the sealed bag. Considering the long-time storage, there's no permission for contamination, chemicals or meteorological variations. From time to time, tubes of each group were extracted to determine the sample recovery. No acrolein was detected in the 10 blank tubes, indicating no contamination during the storage time.

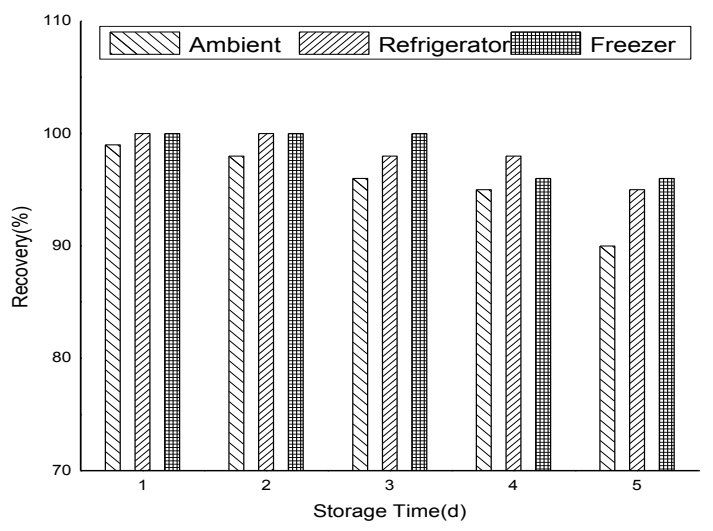

Fig. 4. Storage results for PFPH-coated tubes 


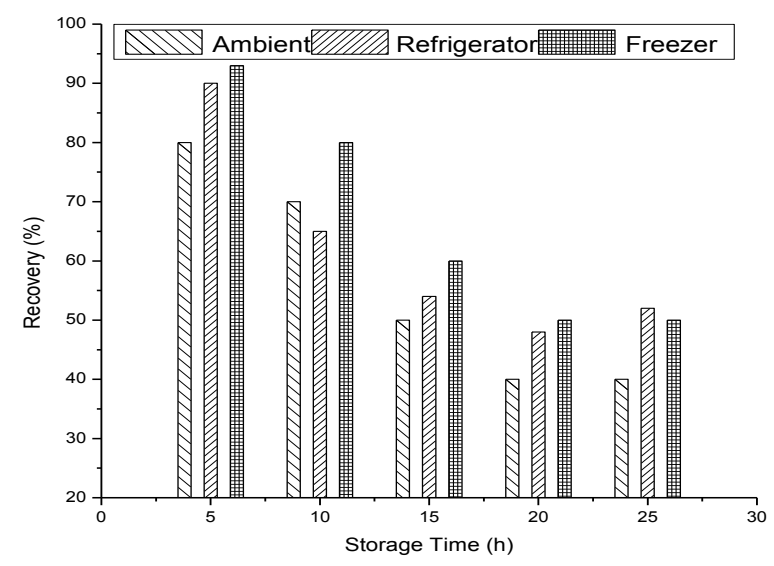

Fig. 5. Storage results for DNPH-coated tubes

For PFPH-coated tubes, Fig.4 shows the recovery keeps above $90 \%$ of the three conditions. Fig. 5 shows the results obtained from 15 DNPH-coated tubes stored for 2 days. In this case, storage would cause decomposition of DNPH-ACR within $5 \mathrm{~h}$. After $15 \mathrm{~h}$, DNPH-ACR decreased rapidly while other adducts were detected by HPLC/UV. DNPH-coated tubes showed a weak storage performance due to the instability of DNPH-ACR.

\section{CONCLUSION}

In this study, two kinds of sampling tubes were developed for the analysis of acrolein in ambient air. Sampling performances of both the PFPH-coated and DNPH-coated silica gel-filled glass tubes were optimized. According to the comparison of the results, the extraction efficiency of PFPH-coated tubes is above $96 \%$. The collection efficiency is over $98 \%$ with the PFPH coating amount of $0.4 \mathrm{mg}$ per $200 \mathrm{mg}$ silica gel(per tube) and the sampling flow rate of $300 \mathrm{~mL} / \mathrm{min}$, the sample storage time can be as long as 5 days without obvious contamination or decomposition. While the extraction efficiency of DNPH-coated tubes is above $92 \%$, the collection efficiency is over $98 \%$ with the DNPH coating amount of $0.3 \mathrm{mg}$ per $200 \mathrm{mg}$ silica gel(per tube) and the sampling flow rate of $800 \mathrm{~mL} / \mathrm{min}$. However, these tubes were inefficient in terms of sample storage due to further formation of other adducts. Above all, the PFPH-coated tubes can be successfully applied in both indoor and field sampling, the DNPH-coated tubes are more suitable for short-time, higher concentration sampling with immediate analysis.

\section{ACKNOWLEDGEMENT}

The authors gratefully acknowledge the financial support from Nanjing University of Science and Technology.

\section{REFERENCE}

[1] Michael C. McCarthy, Theresa E. O'Brien, “Characterization of the Chronic Risk and Hazard of Hazardous Air Pollutants in the United States Using Ambient Monitoring Data," Environ. Health Perspect. , Vol. 117, pp. 791-796, 2009.

[2] Lijuan Wang, Yingxian Sun, “Acrolein, an Environmental Toxin, Induces Cardiomyocyte Apoptosis via Elevated Intracellular Calcium and Free Radicals," Cell Biochem. Biophys., Vol. 61, pp. 131-136, 2011.

[3] Vincent Y. Seaman, Deborah H. Bennett, Thomas M. Cahill, "Indoor acrolein emission and decay rates resulting from domestic cooking events," Atmos. Environ., Vol. 43, pp. 6199-6204, 2009.

[4] Buddhdeb Ghosh, Jiho Park, Katie C. Anderson, Simon W. North, "OH initiated oxidation of 1,3-butadiene in the presence of O2 and NO,” Chem. Phys. Lett., Vol. 494, pp. 8-13, 2010.

[5] Scott A. Steinmetz, Jason S. Herrington, "Crude glycerol combustion: Particulate, acrolein, and other volatile organic emissions,” P. Combust. Inst., Vol. 34, pp. 2794-2757, 2013.

[6] Jason S. Herrington, Michael D. Hays, "Concerns regarding 24-h sampling for formaldehyde, acetaldehyde, and acrolein using 2,4-dinitrophenylhydrazine (DNPH )-coated solid sorbents ," Atmos. Environ., Vol. 55, pp. 179-184, 2012.

[7] Shigehisa Uchiyama, Yohei Inaba, Naoki Kunugita, "Determination of acrolein and other carbonyls in cigarette smoke using coupled silica cartridges impregnated with hydroquinone and 2,4-dinitrophenylhydrazine,” J. Chromatogr. A, Vol. 1217, pp. 4383-4388, 2010.

[8] Jian Li, Yan Li Feng, Chun Juan Xie, "Determination of gaseous carbonyl compounds by their pentafluorophenyl hydrazones with gas chromatography/mass spectrometry," Anal. Chim. Acta Vol. 635, pp. 84-93, 2009.

[9] Xiaobing Pang, Alastair C. Lewis, Jacqueline F. Hamilton, "Determination of airborne carbonyls via pentafluorophenylhydrazine derivatisation by GC-MS and its comparison with HPLC method," Talanta,Vol. 85, pp. 406-414, 2011. 